\title{
Left ventricle outflow tract vegetation, embolism and troponin rise: an infective endocarditis case report
}

\author{
Fortunato Iacovelli • Pietro Scicchitano • \\ Domenico Zanna · Vito Marangelli • \\ Stefano Favale
}

Received: 22 November 2011/ Accepted: 22 January 2012/Published online: 5 February 2012

(C) SIMI 2012

\section{Introduction}

Infective endocarditis (IE) incidence increases with advancing age [1], and recently has often shown atypical onset and poor prognosis. The clinical history varies greatly in relation to the different initial clinical manifestations, possible underlying heart disease, the microorganisms involved, the presence of complications and patient characteristics. Nowadays, the "classic textbook signs" [2] may be found almost exclusively in developing countries. In general, the most common expression of IE (20-50\% of patients [2]) is often an embolic phenomenon. However, acute coronary syndrome (ACS) is an unusual IE onset form, and, as our case seems to describe, is associated with other particular features in the potential clinical evolution of IE.

\section{Case report}

An 85-year-old man was referred from the emergency department to our coronary intensive care unit with a provisional diagnosis of "troponin rise and left ventricular dyssynergy in a hyperpyretic and drowsy patient" (II degree Bricolo's coma scale, temperature: $37.5^{\circ} \mathrm{C}$ ). His only cardiovascular risk factor was hypertension. Since 2003 , there was a history of moderate calcific aortic valve steno-insufficiency, worsened in October 2008 when the last echocardiographic assessment highlighted restricted

F. Iacovelli $(\varangle) \cdot$ P. Scicchitano · D. Zanna · V. Marangelli ·

S. Favale

Section of Cardiovascular Diseases, Emergency and

Transplantations Department, "Aldo Moro" University,

Piazza Giulio Cesare 11, 70124 Bari, Italy

e-mail: fortunato.iacovelli@teletu.it cusp opening with moderate-to-severe regurgitation, but preserved left ventricle ejection fraction (LVEF). Our patient was reported to have been asymptomatic, but exercise testing, useful for unmasking symptoms and in risk stratification [3], was not performed. Probably considering that in asymptomatic patients with severe aortic regurgitation and normal LVEF, the number of events during follow-up is low [3], he was not referred for valve replacement. In May 2008, he had undergone bicameral pacemaker implantation (trifascicular block) in a left subclavian pocket; the pulse generator was later explanted due to pocket infection, and a new right-sided subclavian implantation was performed in 2009. Almost 1 year after device repositioning, the patient's relatives reported persistent, purulent secretions from the left subcutaneous pocket, despite daily medication until a few weeks prior to admission. He had complained of widespread joint pain, asthenia, ankle edema and a temperature of $40^{\circ} \mathrm{C}$ the previous day. The first laboratory test results showed: troponin I $52.21 \mathrm{ng} / \mathrm{ml}$, myoglobin 8,917 ng/ml, GlutamateOxaloacetate Transaminase 438 U/l, Glutamate-Pyruvate Transaminase $91 \mathrm{U} / \mathrm{l}, \mathrm{N}-\mathrm{Terminal}$ pro-Brain Natriuretic Peptide 29,690 pg/ml, C-reactive protein $243.3 \mathrm{mg} / \mathrm{l}$, hemoglobin $11.5 \mathrm{~g} / \mathrm{dl}$, leukocytes $18.06 \times 10^{3} / \mu 1$, neutrophils $91.5 \%$, platelets $50 \times 10^{3} / \mu \mathrm{l}$, fibrinogen $452 \mathrm{mg} / \mathrm{dl}$, D-dimers $14,370 \mu \mathrm{g} / \mathrm{l}$, and also the marks of acute renal failure (urea $229 \mathrm{mg} / \mathrm{dl}$, creatinine $4.01 \mathrm{mg} / \mathrm{dl}$, urine specific gravity 1,030 , hemoglobinuria $0.20 \mathrm{mg} / \mathrm{dl}$, proteinuria $250 \mathrm{mg} / \mathrm{dl}$ and leucocyturia $500 \mathrm{Leu} / \mu \mathrm{l}$ ).

On admission, the blood pressure was $60 / 30 \mathrm{mmHg}$; he was tachypnoeic and tachycardic without a regurgitant heart murmur. A general physical examination did not show specific findings except for an almost complete necrosis of the right thumb (Fig. 1). In spite of oxygen therapy, arterial blood gas analysis suggested a compensated metabolic acidosis: $\mathrm{pH}$ 7.397, $\mathrm{pO}_{2} 90.6 \mathrm{mmHg}$, 


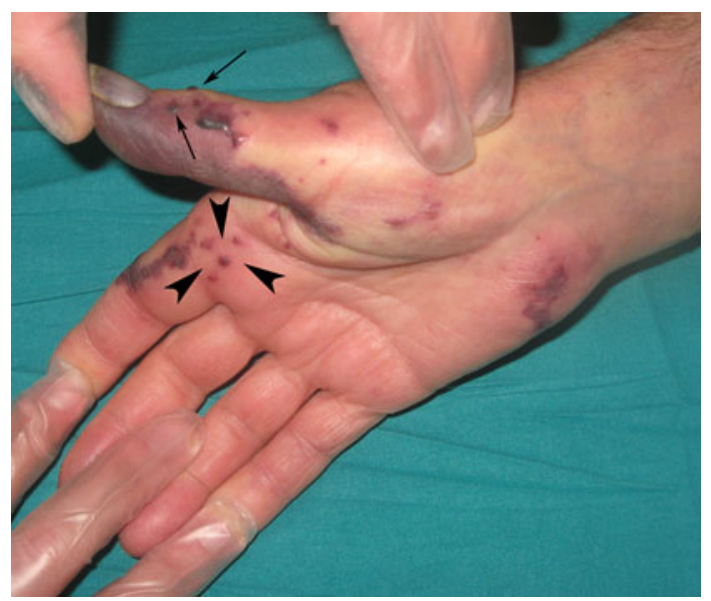

Fig. 1 Purple cyanosis of the ischemic necrotic thumb, petechiae, hemorrhagic bullae, Osler nodes (arrows) and Janeway lesions (arrow heads)



Fig. 2 Cerebral Computed Tomography. Ischemic vascular lesion of recent onset (arrow) in the left frontoparietal region

$\mathrm{pCO}_{2} 30.1 \mathrm{mmHg}, \mathrm{SO}_{2} 97.8 \%, \mathrm{HCO}_{3}^{-} 18.7 \mathrm{mmol} / \mathrm{l}$. An electrocardiogram showed new-onset atrial fibrillation (average ventricular rate $110 \mathrm{bpm}$ ), complete right bundle branch block, and left ventricular hypertrophy. An emergency brain CT scan, performed without contrast medium, revealed a shaded area of cortico-subcortical hypodensity in the left frontoparietal region, consistent with a recent-onset ischemic lesion (Fig. 2). A transthoracic two-dimensional and three-dimensional echo-Doppler, performed using a Philips IE33 sonograph, showed a mobile mass in the Left Ventricle Outflow Tract (LVOT), of a maximum length of $1 \mathrm{~cm}$, consistent with endocardial vegetation. It also highlighted multiple small masses on the mitral leaflets and chordae tendineae as well as voluminous hyperechoic masses on the aortic valve leaflets, consistent with fibrocalcifications or vegetating masses. No signs of right to left shunt were identified, and the measured LVEF was $40 \%$ (Fig. 3).

Hydration and inotropic (norepinephrine, dopamine) therapy was administered, adding furosemide, dexamethasone, paracetamol and an empiric therapy with ampicillin/ sulbactam and vancomycin. Despite a temporary increase in diuresis and normalization of temperature, the patient died 3 days later. The results of blood cultures, available only postmortem, were positive for Staphylococcus aureus.

\section{Discussion}

Staphylococcus aureus was the bacterial pathogen responsible for our IE case, perhaps caused by contamination after device implantation or repositioning. The patient showed troponin increase and left ventricular dyssynergy: it seemed to be an ACS, but, unfortunately, no coronary angiography could be performed because of the patient's adverse clinical conditions and laboratory test results. Coronary embolism was the most likely cause, although we should also consider possible coronary inflammation, which can activate hemostasis and the coagulation cascade, as well as reduced coronary perfusion due to aortic steno-regurgitation, and septic shock from which the patient suffered. Even a myocardial necrosis markers increase may not necessarily be the consequence of an acute myocardial infarction: myocarditis linked to endocarditis, acute heart failure, septic state, acute renal failure and cerebral injuries are well known causes of increased troponin levels that are unrelated to ACS, although myocardial infarction may develop in $9 \%$ of patients with active IE [4].

Another interesting aspect is the localization of endocardial vegetations as the LVOT is not an area in which endocarditis frequently develops. There is limited data in the literature [5] about this implantation site, thus highlighting the uniqueness of our case (Fig. 3) in that the mass grew in the LVOT in spite of a high blood flow velocity. It is quite difficult to explain why. Aortic stenosis and regurgitation may have contributed to damaging the LVOT endocardium over the years, or the hemodynamic instability caused by sepsis favored lesions of the endocardial surface initially, followed by the expression of chemokines, and finally the growth of vegetation. The jet lesion created in this area of the heart induced embolization, precisely in relation to the highest tangential forces created by the blood pulled into the aorta. This localization, therefore, justifies the clinical picture of the patient, especially the widespread embolism in his body, ranging from the brain to the kidneys and perhaps to the coronary arteries. 
Fig. 3 Transthoracic twodimensional $(\mathbf{a}, \mathbf{b})$ and live/realtime three-dimensional (c, d) echocardiography. Septal implantation of the mobile mass (arrows) in the outflow tract of the left ventricle


Moreover, septic embolism certainly could have been the cause of the uncommon injuries on his right hand. In fact, as Fig. 1 shows, he had a well demarcated area of purple cyanosis secondary to epidermal necrosis extending from the distal to the proximal edge of the thumb; petechiae (some of which were coalescing into hemorrhagic bullae); violaceous-reddish, hemorrhagic, infarcted, palpable nodules on the dorsal and lateral surfaces of the thumb consistent with Osler nodes; and purple-red, macular, papular, infarctive lesions on the palm and surface of the thumb and index fingers consistent with Janeway lesions.

In conclusion, the uniqueness of this IE case report lies in the unusual location of the vegetation, and in the rarity of such findings in our developed countries.

Acknowledgments We would like to thank Dr. Rosalind Lee for her excellent help in the English revision of the manuscript. We would like to thank the neuroradiologists of the University of Bari in the adaptation of the images. Finally, we would like to thank Dr. Doriana Apruzzi for the dermatological analysis of the patient's lesions.

Conflict of interest None.

\section{References}

1. Hoen B, Alla F, Selton-Suty C, Association pour l'Etude et la Prévention de l'EndocarditeInfectieuse (AEPEI) Study Group et al (2002) Changing profile of infective endocarditis: results of a 1-year survey in France. JAMA 288(1):75-81

2. Habib G, Hoen B, Tornos P, ESC Committee for Practice Guidelines et al (2009) Guidelines on the prevention, diagnosis, and treatment of infective endocarditis (new version 2009): the Task Force on the Prevention, Diagnosis, and Treatment of Infective Endocarditis of the European Society of Cardiology (ESC). Endorsed by the European Society of Clinical Microbiology and Infectious Diseases (ESCMID) and the International Society of Chemotherapy (ISC) for Infection and Cancer. Eur Heart J 30:2369-2413

3. Vahanian A, Baumgartner H, Bax J, ESC Committee for Practice Guidelines et al (2007) Guidelines on the management of valvular heart disease: the Task Force on the Management of Valvular Heart Disease of the European Society of Cardiology. Eur Heart J 28:230-268

4. Demin AA, Drobysheva VP (2004) Myocardial infarction in patients with infectious endocarditis. Kardiologiia 44(1):4-9

5. Quah BS, Selladurai BM, Jayakumar CR, Mahendra Raj S (1993) Left ventricular outflow tract (LVOT) vegetations and spontaneous obliteration of a large ruptured intracranial mycotic aneurysm in a case of infective endocarditis. Singapore Med J 34(2):172-174 